\title{
Adolescencia y criminalidad en el sistema penal colombiano: un estudio desde la noción de responsabilidad subjetiva*
}

\section{Adolescence and criminality in the Colombian penal system: a study from the notion of subjective responsibility}

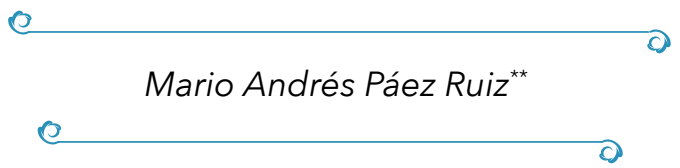

Recibido 01.11. 2017 • Arbitrado 13.12.2017•

Aprobado 12.01. 2018

Este estudio se encuentra vinculado a la línea de Políticas Publicas del grupo de investigación Ciencia Política del Instituto de Estudios Políticos de la Universidad Autónoma de Bucaramanga. Se agradece especialmente la corrección de estilo realizada por la Dra. María Eugenia Bonilla y la Dra. Lorena Arraiz.

* Psicólogo Especialista en Psicología Clínica y de la Salud, Magíster en Ciencia Política, Profesor en la Facultad de Ciencias de la Salud de la Universidad Autónoma de Bucaramanga, mpaez2@unab.edu.co

\section{Resumen}

Entendiendo la ciencia política en la perspectiva de Manuel Alcántara como un análisis de sistemas políticos, y reconociendo en esta ciencia social un terreno de investigación multidisciplinaria, el presente estudio corresponde a un análisis elaborado con las categorías definidas como Polity, Policy y Politics, aplicadas a las políticas de infancia y adolescencia vigentes en Colombia. Para tales efectos, el proceso metodológico implicado en este trabajo, se encuentra apoyado en los aportes conceptuales del psicoanálisis y en las orientaciones metodológicas ilustradas por el filósofo francés Michel Foucault. El objetivo de esta investigación, obedece al interés por indagar acerca del lugar que le otorga la Ley 1098 de 2006 a la noción de responsabilidad, en el marco del Sistema Colombiano de Responsabilidad Penal para Adolescentes (SRPA). Por consiguiente, se han analizado las disposiciones y fundamentos de dicha ley, con el fin de interrogar la implementación de esta última a partir de la noción de Responsabilidad Subjetiva, en referencia a la relación entre adolescencia y crimen.

Palabras clave: Sistemas políticos, adolescencia, criminalidad, responsabilidad subjetiva 


\section{Abstract}

Understanding political science in the perspective from Manuel Alcántara as an analysis of political systems, and recognizing in this social science a field of multidisciplinary research, the present study corresponds to an analysis elaborated with the categories defined as Polity, Policy and Politics, applied to the policies of childhood and adolescence in force in Colombia. For these purposes, the methodological process involved in this work, is supported by the conceptual contributions from psychoanalysis and from the methodological orientations illustrated by the French philosopher Michel Foucault. The objective of this investigation is due to the interest of inquire the place granted by Law 1098 of 2006 to the notion of responsibility, within the framework of the Colombian System of Criminal Responsibility for Adolescents (SRPA). Consequently, the legal provisions and foundations of this law have been analyzed, in order to interrogate the implementation of the latter from the notion of Subjective Responsibility, referring to the relationship between adolescence and crime.

Key words: Political systems, adolescence, crime, subjective responsibility.

\section{Introducción}

Entre 1930 y 1940 en la filosofía jurídica anglosajona se produjo una transición del formalismo legal al realismo jurídico. Desde entonces, la formulación de disposiciones legislativas tomaron como referencia primordial el conocimiento aportado por las ciencias sociales (Soria, 1998). En consideración de tal premisa, la noción de niñez y adolescencia del Sistema Colombiano de Responsabilidad Penal para Adolescentes (SRPA) se espera sea consistente y consecuente con los diversos abordajes que desde disciplinas como la psicología, la psiquiatría, la sociología y la antropología, se proponen al respecto.

Sin embargo, una revisión al interior de dichos sistemas de conocimiento pone de manifiesto que las nociones mencionadas resultan notoriamente polisémicas y con definiciones controvertidas entre sí (Casco y Oliva, 2004). En este punto, se abre la posibilidad investigativa acerca de cuáles son las nociones de niñez y de adolescencia que fundamentan la Ley 1098 de 2006, y por consiguiente en el Sistema de Responsabilidad Penal para Adolescentes en Colombia.

En Colombia el sistema legislativo aplicado en niños, niñas y adolescentes permite inferir el uso de algunos postulados de la psiquiatría forense, de la ciencia del comportamiento y de la psicología del desarrollo, para atribuir a 
partir de los mismos la inimputabilidad del menor de edad según tres factores puntuales a saber: a) las condiciones dependientes del estado de salud mental del adolescente (Alarcón y Vidal, 1986); b) la influencia causal de contingencias ambientales (socioeconómicas) sobre el comportamiento del adolescente (Skinner, 1971, 1974) y; c) las posibles limitaciones cognitivas y afectivas relativas al juicio y al criterio moral del adolescente (Perinat, 1998).

En el proceso de revisión de esta teoría del delito, surge el interés de indagar sobre los fundamentos epistémicos de tales atribuciones. En una primera mirada acerca de este asunto, sobresale la circunstancia de que dichas explicaciones que derivan de los dominios de la biomédica, el ambientalismo y la psicología, no ofrecen suficiente claridad al respecto de las condiciones en que estos saberes de tan diferente orientación, e incluso en ciertos aspectos excluyentes entre sí, se articulan en una misma teoría del delito, que sirve, a su vez, para fundamentar una práctica legislativa ejercida a través del Sistema de Responsabilidad Penal para Adolescentes.

De allí que el propósito de esta investigación sea indagar acerca del lugar que le otorga la Ley 1098 de 2006 a la noción de responsabilidad en el Sistema Colombiano de Responsabilidad Penal para Adolescentes, mediante la elaboración de un análisis discursivo del contenido y los fundamentos de dichas disposiciones legislativas, con el fin de precisar la función de la subjetividad en la comprensión de la relación política entre adolescencia y crimen.

Para ello se plantea el alcance de tres objetivos específicos. El primero, es describir las "condiciones de emergencia" a partir de las cuales se definen las nociones de niñez y adolescencia en el SRPA. El segundo, es interrogar la episteme que fundamenta el vínculo formalmente establecido entre adolescencia y crimen en el Sistema Colombiano de Responsabilidad Penal para Adolescentes. El tercero pretende formalizar los alcances analíticos de la noción de responsabilidad subjetiva aplicados en una reflexión acerca de la relación política entre adolescencia y crimen.

La pertinencia del problema de investigación delimitado en este estudio, estriba en el interés comprendido actualmente por las discusiones tanto académicas como profesionales sobre los fundamentos políticos de la responsabilidad penal del adolescente infractor, y asimismo, sobre las indagaciones necesarias al respecto de los conceptos básicos implicados en el sistema colombiano de responsabilidad penal para adolescentes. Los dilemas planteados por la jurisprudencia de este sistema y sus propósitos pedagógicos dirigidos al menor de edad atendido, indican la premura de interrogar las concepciones que subyacen legitimadas por el Estado a través de las políticas de infancia y 
adolescencia, en los discursos que formulan las relaciones entre las nociones de adolescencia, responsabilidad y crimen.

A la luz de dicha pertinencia, la razón que justifica la viabilidad en la elaboración de la presente investigación, radica en el carácter multidisciplinario de su respectivo planteamiento. Esta manera de investigar, se entiende como la formulación de proyectos cuyos resultados deriven de un dialogo que integre diversas miradas disciplinares o metodológicas al respecto de un mismo fenómeno.

En el caso de este estudio, dicha multidisciplinariedad, tiene como punto de partida la posibilidad de integración que ofrece la concepción de ciencia política en tanto análisis de sistemas políticos (Alcántara Sáez, 1993). Al abordar las políticas de infancia y adolescencia a partir de las categorías de análisis definidas como Polity, Policy y Politics, tal ejercicio justifica la inclusión de las ideas comprendidas por Foucault (1969) acerca de lo que éste define como un análisis discursivo, al igual que justifica la inclusión de la teorización psicoanalítica, especialmente en razón de lo concerniente a los asuntos relativos a la Politics.

\section{Precisiones metodológicas}

El proceder de esta investigación obedece a la realización de un análisis discursivo, tomando como referencia para su definición el trabajo presentado por el filósofo francés Michel Foucault (1969), quien señala que un análisis discursivo no subyace a un protocolo o un procedimiento estandarizado que se replica indiscriminadamente en uno y otro trabajo, sino que representa una serie de hipótesis que orientan la forma de leer y comprender en un discurso la singularidad de las estrategias, las formaciones y las condiciones que hacen posible su existencia.

En este sentido, el discurso no es asumido simplemente como un contenido expresado en un "decir" o en la formalidad de un documento, sino como la manifestación de una relación de poder ubicada en una época y un contexto determinados, ejercida mediante el saber - poder sobre el estilo de vida y las prácticas de los sujetos implicados, en relación con un dominio de conocimiento, una forma de gobierno o el establecimiento de algún tipo de control sobre la vida cotidiana (Foucault, 1969).

La ciencia política y el psicoanálisis freudiano han compartido escenarios de dialogo en los cuales autores reconocidos en la ciencia política tales como David Roll, han reconocido el valor de los aportes conceptuales, teóricos y 
metodológicos legados por Sigmund Freud para el abordaje de temáticas tan complejas como el orden del poder político, la psicología de las masas y el problema de la legitimidad (Roll, 2011).

En virtud de dicha cercanía entre la ciencia política y el psicoanálisis, como también en razón del interés que atraviesa este estudio por la subjetividad, se ha considerado pertinente implementar en esta labor dos de los principios que orientan la investigación psicoanalítica aplicada en el estudio de fenómenos sociales; a saber, el principio de la docta ignorancia y el principio de la precisión (Gallo y Ramírez, 2012). La pertinencia de estos dos principios fue haciéndose cada vez más evidente mientras se avanzaba en la realización de este trabajo, dado el análisis de la relación entre un referente normativo y un sujeto de compleja profundidad psíquica.

Tomado de la mayéutica socrática, pero también implementado en las prácticas de la clínica y la investigación psicoanalítica (Gallo y Ramírez, 2012), el principio de la docta ignorancia enseña que ningún conocimiento se encuentra exento de ser sujetado a la duda y el escrutinio riguroso. De esta manera, el investigador no da por hecho que tiene un saber solido o incuestionable por muy obvio, popular o aceptable que éste parezca. El investigador orientado por este principio permanece en una posición constante de interrogación y aprendizaje ante sus objetos de estudio, es decir, no asume la posición del que no necesita ser enseñado por el fenómeno que estudia, sino que hace de cada aproximación a su fenómeno de interés una experiencia nueva de la que puede derivar un nuevo conocimiento.

En la formulación de la pregunta de investigación que ha ocupado este estudio, la aplicación de este principio en particular fue de vital importancia. Para dar con el planteamiento de dicha pregunta, el investigador ha tenido en primer lugar que renunciar a la seguridad de todo lo que creía conocer acerca de la temática investigada, en consecuencia, elementos relacionados con la misma que han sido a su vez precisados como las fuentes del problema de investigación, fueron revisados sin el prejuicio de que popularmente estos elementos corresponden a cuestiones innecesarias o en su defecto ya resueltas.

En este orden de ideas, el problema de investigación referido, ha resultado del ejercicio de explorar las concepciones políticas y legislativas que explican la responsabilidad penal del menor de edad en Colombia, a partir de una perspectiva que reconoce una posición de "no saber" ante su objeto de estudio. En consecuencia, la experiencia de plantearse un interrogante ante este tema no se resuelve a partir de argumentos de autoridad o saberes reciclados que obturan la investigación, sino mediante la posibilidad de buscar o de hacer un camino particular en la construcción de un saber nuevo que confirme 
lo que ya se conoce, o que también pueda ampliarlo, contradecirlo o transfórmalo si es el caso.

Trabajar sin estándares estadísticos o sin intervenir en una muestra de sujetos de investigación no denota una labor que carezca de rigurosidad de carácter científico ni de un dispositivo metodológico que oriente el análisis. Un fenómeno social es susceptible de ser investigado con rigurosidad al ser asumido como una práctica discursiva; esta categoría metodológica, tomada del filósofo francés Michel Foucault, hace referencia a la comprensión de un acontecimiento $\mathrm{u}$ objeto de estudio que toma forma por efectos de un discurso, entendido este último como manifestación del poder que eventualmente puede ejercer un sistema de gobierno o un sistema de conocimiento (Foucault, 1970).

$\mathrm{Al}$ trabajar, entonces, con prácticas y formaciones discursivas es necesario precisar con cuidado y rigurosidad dos aspectos concretos en relación con la temática investigada. En primer lugar, la definición de los criterios de inclusión del material que será asumido como objeto de análisis y; en segundo lugar, la revisión del detalle en el análisis conceptual que tendrá lugar mediante los objetivos del estudio planteados.

En el caso particular de esta investigación, considerando los límites demarcados por la pregunta que ha sido formulada, el material tomado para el análisis respectivo corresponde a los documentos oficiales en los cuales se formalizan las políticas de infancia y adolescencia vigentes en Colombia, específicamente decretados desde el año de 1991 hasta el año 2017. La demarcación de este periodo de tiempo obedece a que es desde la constitución política de 1991 cuando se comienzan a identificar cambios políticamente significativos en relación a las disposiciones en cuestión. Asimismo, son importantes en esta investigación los artículos o informes estatales publicados en los límites de este periodo de tiempo, que reportan cualquier estudio realizado que pueda ofrecer hallazgos relevantes o descripciones relativas al avance, los cambios y los efectos de las políticas de infancia y adolescencia aplicadas en Latinoamérica y específicamente en Colombia.

Cuando se hace alusión a un análisis discursivo referenciando para ello el trabajo de Michel Foucault (1969), se alude no a una técnica de interpretación sino a una forma de leer un acontecimiento social, político, económico o epistémico, que ha tomado forma según las condiciones dadas en un momento histórico específico y esencialmente por las vías del discurso. Ahora bien, en esta perspectiva, aunque un discurso no deja de tener una formalización material que se expresa en el "decir" de una ideología, un documento oficial, un archivo, o un dominio de conocimiento, la lectura del mismo no 
debe restringirse al contenido literal de tal formalización, ya que los discursos implican relaciones de poder que pueden ser rastreadas más allá de lo que es evidente en ellos a simple vista.

Foucault (1969) advierte mediante el análisis retrospectivo de su propia obra, acerca de unas hipótesis iniciales a considerar en la lectura de un discurso; unas hipótesis por las que el investigador puede comenzar a interrogar la procedencia y los efectos del discurso que han dado forma a su objeto de estudio. De esta manera, llegando un poco más allá de aquello que manifiesta explícitamente un discurso, Foucault (1969) abre la posibilidad de hacer preguntas sobre las condiciones históricas que hacen posible la emergencia, proliferación y predominancia de un discurso, también acerca de cómo un discurso se sirve de diversos dominios de conocimiento sin llegar a sujetarse a los parámetros epistemológicos de tales dominios y cómo un discurso da lugar a la existencia de objetos, conceptos y enunciados que se convierten en instrumentos para el ejercicio de un poder que bien puede ser político, económico o de conocimiento.

\section{El menor de edad en el sistema colombiano de}

\section{responsabilidad penal para adolescentes}

El primer aspecto que debe ser esclarecido para dar respuesta a la pregunta de investigación que ocupa este estudio, consiste en precisar las nociones de infancia y adolescencia enunciadas en la Ley 1098 de 2006 y en el SRPA. Según estas disposiciones legislativas, la función de la noción de responsabilidad se encuentra limitada por aquello que el Estado colombiano reconoce políticamente como un menor de edad.

Definir con precisión las nociones de infancia y adolescencia, no solo representa una labor compleja debido a la multiplicidad de factores que deben ser considerados y ordenados al respecto, sino que también implica lidiar con una controversia densamente documentada entre los sectores de las ciencias naturales y las ciencias sociales que se han interesado en el estudio del tema (Casco y Oliva, 2004). En esta controversia, algunas posiciones afirman que el factor determinante en tales definiciones es de naturaleza biológica, mientras otras sostienen que dicho factor es completamente cultural. Los resultados conceptuales que derivan de estos debates acaban por servir de referencia a los campos de formalización y legitimación de la legislación y del ejercicio del derecho. 
Un concepto relevante al que ha sido posible llegar es a la categoría denominada como "menor de edad". En la ley 1098 de 2006 y en el SRPA, este término se usa como una categoría formal que enuncia al sujeto que se hace objeto de la existencia y aplicación de estas dos disposiciones. También destaca el hecho de que tal categoría agrupa en un mismo concepto tanto a niños como adolescentes, sin hacer mayores distinciones al respecto de las particularidades susceptibles de ser señaladas en materia de infancia y adolescencia.

Por otra parte, está el asunto de las implicaciones políticas y filosóficas comprometidas en la enunciación del concepto moderno de "menor de edad". Es el principio kantiano de "supere aude", el que introduce y establece en la modernidad el concepto de "mayoría de edad", y el mismo que por contra versión, introduce también el concepto de "minoría de edad". Es importante reconocer que en sus planteamientos originales, el límite entre estas dos condiciones no se encuentra determinado por la madurez cronológica o por un umbral marcado en años de vida, sino como Kant lo explica, la mayoría de edad hace aparición cuando el ser humano es libre, valiente, capaz y decidido para pensar por sí mismo y haciendo uso de su propio entendimiento. En contra versión a esto, la minoría de edad es entonces una condición devenida principalmente de la pereza y de la cobardía que impulsan a alguien a dejar que un libro reemplace su criterio propio, un director espiritual reemplace su conciencia moral, y un médico direccione su régimen de cuidado y su dieta (Kant, 1784, ed. de 1998:18).

Immanuel Kant, con sus "Críticas" dedicadas a la razón, al juicio y a la moral, como también con sus disertaciones sobre la ilustración dadas a conocer durante la segunda mitad del siglo XVIII, fue el filósofo quien dio forma a los referentes del discurso sobre la modernidad (Foucault, 1994). Durante el siglo de las luces, los avances tecnológicos, las grandes luchas sociales, la filosofía política y los nuevos paradigmas epistemológicos, se vieron movilizados y atravesados por las ideas del racionalismo kantiano y por la revolución ideológica de la ilustración, esto trajo consigo la aparición de las sociedades liberales y de las teorías modernas del derecho. La ley 1098 de 2006 y el SRPA, presuntamente funcionan con las mismas lógicas modernas que rigen en las sociedades liberales, por tal razón, llama la atención que agrupen a niños y adolescentes en la categoría de menores de edad, asumiendo que esta es una condición definida por la edad cronológica tal y como es posible de leer en el siguiente artículo:

Ley 1098 de 2006, Artículo 3 "Para todos los efectos de esta ley son sujetos titulares de derechos todas las personas menores de 18 años. Sin perjui- 
cio de lo establecido en el artículo 34 del Código Civil, se entiende por niño o niña las personas entre los 0 y los 12 años, y por adolescente las personas entre 12 y 18 años de edad" (CRC, 2006:35).

PARÁGRAFO 1o. "En caso de duda sobre la mayoría o minoría de edad, se presumirá esta. En caso de duda sobre la edad del niño, niña o adolescente se presumirá la edad inferior. Las autoridades judiciales y administrativas, ordenarán la práctica de las pruebas para la determinación de la edad, y una vez establecida, confirmarán o revocarán las medidas y ordenarán los correctivos necesarios para la ley" (CRC, 2006:35).

El rastreo de esta categoría denominada minoría de edad a la que hace alusión la ley en cuestión y el sistema en ella consagrado, indica la enunciación y los efectos de una formación discursiva, orientada por la pretensión no de describir la condición natural propia de un niño o de un adolescente, sino de nombrar y hacer aparecer al niño y al adolescente como sujetos de una condición de déficit y de impotencia (física y mental), que se ratifica con la idea de población vulnerable y de recurso adaptable en la que se asume la infancia y la adolescencia en Colombia. De hecho, las pruebas que menciona el artículo para ser aplicadas en casos donde la condición de minoría de edad se encuentra puesta en duda, son pruebas de peritaje cuya implementación es asignada a profesionales de la medicina y de la psicología, quienes tienen a su servicio un acervo instrumental, técnico y metodológico dispuesto para hacer aparecer evidencia relativa a las limitaciones y a la dependencia orgánica, cognitiva, emocional y ambiental que en apariencia, constituye lo esencial en niños y adolescentes.

Las políticas de infancia y adolescencia han sido presentadas en Latinoamérica como reformas de los sistemas legislativos realizadas en favor de la protección de los menores de edad, al igual que como políticas de Estado orientadas a garantizar los pactos internacionales concernientes a los derechos de los niños y los adolescentes (Ministerio de Desarrollo Social de la Nación et al., 2008; Berríos Díaz, 2011). Ahora bien, un análisis detallado de las mismas, con el cual sea posible abordar más su contenido político que sus disposiciones jurídicas, pone al descubierto que tales políticas son también estrategias que movilizan o sostienen los intereses particulares de ciertos sistemas de gobierno. 


\section{Análisis del vínculo epistémico entre adolescencia y}

\section{criminalidad en el sistema de responsabilidad penal para}

\section{adolescentes}

La relación entre adolescencia y criminalidad no es un fenómeno natural por medio del cual una de ellas es inherente a la otra, por el contrario, esta asociación ha sido el producto de un estereotipo generalizado que ha tomado forma en el discurso de las ciencias naturales y sociales, para justificar la atribución que en escenarios pedagógicos y familiares se ha usado para explicar el comportamiento "problemático" de los adolescentes (Casco y Oliva, 2004). Así pues, esta relación entre adolescencia y criminalidad ha servido al planteamiento y sostenimiento del actual Sistema colombiano de Responsabilidad Penal para Adolescentes.

Iniciando la década de 1990, los efectos urbanos de la guerra del Estado contra los carteles del narcotráfico, las difíciles condiciones de subsistencia económica derivadas de los altos índices de desempleo, y la injusticia socioeconómica, que impedía el acceso a salarios dignos para las clases sociales más desfavorecidas, fueron provocando una acelerada vinculación de niños y adolescentes a las maquinarias de la delincuencia común y del crimen organizado en Colombia. En las actividades del sicariato, del micro tráfico de estupefacientes, y del hurto a pequeña y gran escala, una notable población de menores de edad encontró una opción de vida económicamente redituable, al tiempo que reconocían una forma segura de sobrevivir y de abrirse paso en la pirámide social de un país tradicionalmente violento.

Ahora bien, el registro acertado de la situación emergente en los años mencionados, difícilmente se ha logrado captar mediante las estadísticas oficiales recogidas y publicadas desde aquel entonces. Este registro, en la actualidad, puede leerse mejor testificado mediante trabajos de orientación periodística y literaria basados en la investigación cualitativa y el análisis de historias de vida. Consecuentemente, testimonios periodísticos tales como la crónica titulada No Nacimos pa' Semilla, material publicado en el año de 1990 bajo la autoría de Alonso Salazar, o novelas como La Virgen de los Sicarios, publicada en 1994 bajo la autoría de Fernando Vallejo, o Rosario Tijeras de Jorge Franco publicada en 1999, empezaron a dar forma de manera contundente y principalmente en el ámbito de la opinión popular, a la idea que delata en el menor de edad una insospechada iniciativa para la ejecución de actos delictivos de diversa índole, actos que iban desde infracciones simples hasta crímenes agravados. 
La imagen de este niño y de este adolescente "violento", corroborada por los muchos casos atendidos en ciudades capitales a través de las instituciones oficiales encargadas, se hizo más sólida alrededor del año 2004 gracias a la situación de reclutamiento de menores en las filas de la militancia armada insurgente. Aunque esta situación se documentó formalmente a partir del año 2007, como efecto de las investigaciones movilizadas por la Ley 975 de 2005 , las cuales fueron encabezadas principalmente por defensores de derechos humanos y activistas sociales pertenecientes a organizaciones no gubernamentales, los relatos de las víctimas de violencia sociopolítica en Colombia denunciaban desde el año de 1990, el entrenamiento y la participación de menores de edad en actos terroristas y en zonas de combate armado entre las fuerzas militares del Estado y grupos del paramilitarismo (Comisión delegada informe COLOMBIA NUNCA MÁS, 2008:114).

En las disposiciones consignadas en el SRPA no es posible encontrar una explicación concreta que fundamente las directrices que excluyen al menor de edad de su responsabilidad penal ante un hecho ilícito en el que se halle sindicado como autor material. Sin embargo, estas mismas directrices ofrecen indicios que permiten rastrear la influencia dominante de paradigmas de orientación ambientalista y biologicista que han propiciado un discurso en el cual existe una asociación de inimputabilidad entre adolescencia y criminalidad.

\section{Teoría Skinneriana de orientación ambientalista}

En las relatorías correspondientes a la jurisprudencia del SRPA, los jueces encargados sugieren que factores tales como la familia y la situación socioeconómica del menor son determinantes a considerar en el estudio de las causas y las sanciones relativas para infractores entre los 12 y los 18 años de edad (Escobar, 2013). Estas afirmaciones oficiales movilizan una serie de procedimientos institucionales que actúan en favor de la confirmación de las mismas; de esta manera, saberes como el de la psicología clínica, la pedagogía y asimismo el de otros dominios de la intervención social y comunitaria, se ponen al servicio del Estado para producir protocolos, enunciados y evidencias orientadas a justificar la causalidad del comportamiento delictivo del adolescente con factores ambientales.

La teoría de orientación ambientalista que más ha contribuido a posicionar la noción de adolescente criminal que se ha visibilizado en el SRPA, corresponde a la filosofía social de B.F. Skinner (1974), también conocida como ciencia del comportamiento. Esta teoría que cobró auge en Norteamérica durante el 
periodo comprendido entre la década de 1950 y 1970, no solo ha tenido una reconocida resonancia en los dominios temáticos de la psicología, sino en las ciencias sociales en general, incluso en la ciencia política (Sanders, 1995).

Skinner fundó la idea de que el comportamiento humano se define en estrictos términos científicos, como un resultado directo o indirecto de un proceso sucedido a partir de contingencias ambientales. La determinación de tales contingencias y su sistematización en programas ordenados garantiza las condiciones necesarias para regular el comportamiento social de un organismo (Skinner, 1974).

La influencia económica y colonizadora de las tendencias políticas e intelectuales del pueblo norteamericano sobre la comunidad internacional, ha sido una circunstancia que ha facilitado la diseminación de las ideas de Skinner (1974), a través de diferentes modelos teóricos y metodológicos relacionados con el orden social y con la gobernanza. Como consecuencia directa, el SRPA promueve la concepción de menor de edad asumiendo que la responsabilidad concomitante de su comportamiento delictivo debe ser penalmente inimputada y correctivamente trasferida al medio familiar, educativo y social del adolescente infractor.

\section{Teoría de orientación biologicista}

Otra teoría que se ha sumado a la construcción de la noción de adolescente criminal posible de rastrear en el SRPA, es la teoría de orientación biologicista que hace énfasis en la explicación orgánica del comportamiento humano. Esta teoría sostiene que el comportamiento humano, y especialmente el comportamiento adaptativo o desadaptativo, es una consecuencia directa de la acción de las funciones fisiológicas, metabólicas, neurológicas o genéticas inherentes al organismo. De esta manera, el comportamiento violento de un adolescente puede ser aceptablemente explicado por la hipótesis de una influencia genética (Jara y Ferrer, 2005). Asimismo, el comportamiento delictivo puede atribuirse a la afectación producida por una enfermedad de naturaleza psiquiátrica (Alarcón y Vidal, 1986).

En el SRPA, la influencia de la teoría de orden biologicista subyace puntualmente en el concepto de menor de edad allí asumido. Los aportes de la psicología del desarrollo de origen norteamericano han afirmado la creencia de que la maduración del sistema nervioso y el sistema endocrino en los seres humanos representa la condición formadora de las posibilidades psicológicas de las que deriva el comportamiento (Perinat, 1998). En este sentido, la minoría de edad, ubicada por debajo del rango de los 18 años, se asume como 
una condición temprana del desarrollo en la cual las facultades psicológicas de un niño y de un adolescente, no poseen el nivel suficiente de maduración para dar cuenta de la determinación, la comprensión y la responsabilidad legal implicadas en un acto delictivo.

\section{Legitimación institucional de la noción de adolescente criminal del SRPA}

Las teorías tanto ambientalista como biologicista, no han conseguido ser los modelos dominantes que explican la relación entre adolescencia y crimen gracias a la suficiencia de sus contenidos. Por el contrario, han sido otras las causas que han otorgado a estas ideas la posición de veracidad de la que han sido envestidas. Si bien, con estas teorías ha aparecido la noción de adolescente criminal, ello ha sido posible solo a partir de la combinación de ciertas condiciones que legitiman su enunciación dentro de un marco institucional y con carácter de autoridad de conocimiento, un fenómeno que acaba por inscribirse como objeto de la legislación nacional y de diversas formas de intervención de tipo clínico y pedagógico.

Cuando el Estado necesita que la sociedad civil legitime una forma específica de gobierno, éste concentra sus esfuerzos en persuadir a la ciudadanía acerca de las ventajas de aceptar un determinado pacto social, que puede ser de carácter legislativo, económico o político. De allí que la legitimidad funciona efectivamente solo a partir de la acción conjunta entre el Estado y la sociedad civil. Este acuerdo entre los funcionarios del gobierno y la población que representan es lo que en la actualidad se denomina gobernanza (Aguilar, 2014), e implica que los sectores públicos y privados aporten recursos de diferente naturaleza en favor y acuerdo con los intereses del gobierno electo. Es entonces a la luz del concepto de gobernanza que es posible comprender cómo se ha hecho aparecer la noción de adolescente criminal, sustentada en teorías ambientalistas y biologicistas.

Para que el Estado colombiano pudiera sostener el SRPA en virtud de sus intereses políticos y económicos, la academia como institución pedagógica y el sector salud como autoridad médica, empezaron a producir recursos tales como estudios experimentales y protocolos de evaluación e intervención, que formalizaron la inscripción del comportamiento delictivo de los adolescentes como conducta desadaptada asociada al trastorno mental, al déficit del desarrollo y a los ambientes adversos, lo cual ratificó el discurso científico al respecto de la noción de adolescente criminal, pronunciada en la literatura psiquiátrica norteamericana. Lo anterior, sirvió como plataforma de encuentro entre los intereses políticos y económicos del Estado para que en la primera 
década del siglo XXI pudieran existir condiciones de invención del adolescente criminal como objeto de la legislación autorizada por las ciencias de la salud.

De esta manera, el SRPA se fundamenta en una concepción de adolescencia que coincide con la que a este mismo respecto, predomina en los documentos políticos que circulan entre la comunidad internacional; documentos que han marcado en los países latinoamericanos la tendencia de hacer uso del concepto de menor de edad en la formulación de sus legislaciones. Por tal razón, este sistema se dirige a un adolescente criminal que no hace uso de su propia palabra, razón y responsabilidad, ya que el Estado y la sociedad civil nombran al infractor en este caso como un objeto desviado, incapaz o trastornado que se hace necesario introducir en un tratamiento de orientación terapéutica, cautelar o preventiva.

Estas medidas asumidas por el Estado colombiano son consecuentes con las disposiciones oficiales de la Organización de Estados Americanos (OEA) y de la ONU, en torno a la situación de vulnerabilidad en la que se categoriza a la población infantil. Dicha línea de coincidencia política tiene por consecuencia favorecer su posición ante el panorama de las relaciones internacionales y, por ende, facilita el curso de tratados económicos. Ante estas consideraciones, es difícil aceptar que el SRPA se fundamente en nociones desarticuladas de la influencia producida por la economía y la política internacional.

\section{Psicoanálisis y responsabilidad subjetiva}

En primer lugar, es necesario señalar que la noción de sujeto a la que el psicoanálisis dedica su interés no es la misma noción de sujeto asumida en el derecho penal o en la psicología forense. Esta noción de sujeto que opera en el psicoanálisis es la que hace referencia a un sujeto de lenguaje que construye su estructura psíquica a partir de las posibilidades de simbolización y significación, ofrecidas precisamente por el lenguaje. Es también aquella que refiere un sujeto capaz de producir un saber particular al respecto de su vínculo con el significante de su cuerpo y acerca de la posición en que sus actos le referencian o dirigen a un "otro" (Lacán, 1966).

En este orden de ideas, dicho sujeto aunque se encuentra caracterizado por un faltante estructural ${ }^{1}$ latente en su psiquismo, no es concebido a partir de la

1 La experiencia clínica que se ha formalizado al interior del psicoanálisis freudiano y lacaniano, ha brindado elementos para comprender la condición humana como un permanente estado de incompletud. Por esto mismo, en el psicoanálisis se nombra al ser humano con la noción de sujeto y no de persona o individuo, como si ocurre en la psicología. Esta noción por la que opta el psicoanálisis, asume a un ser humano que se descubre a sí mismo sujeto a una angustia de castración con la que puede reconciliarse pero de la que nunca podrá deshacerse (Freud, 1929 - 30), asimismo, un ser humano 
imposición del déficit, por tanto, el psicoanálisis no se resiste a leer en el niño o en el adolescente transgresor a un sujeto del derecho con plenas facultades para implicarse en su propio acto y asumir la responsabilidad que le lleva a apropiarse del mismo.

En segundo lugar, está el tratamiento que aplica el psicoanálisis en el rastreo del sentido del acto trasgresor en el niño y en el adolescente. Mientras que la intervención de la psicología forense ofrece una interpretación objetiva acerca del acto trasgresor, en la que se registra la evidencia que sirve en una formulación diagnóstica de tipo clínico o pericial, el psicoanálisis por otra parte, procura proponer las condiciones para que acontezca una producción subjetiva que permita reconstruir, en retrospectiva, el sentido particular del acto trasgresor. En esta posición, el psicoanálisis no se interesa por la búsqueda de un diagnóstico forense, más bien, podría decirse que usa todos sus recursos metodológicos para orientar al sujeto en la interrogación propia acerca de sus implicaciones en el acto del cual éste puede responder.

En esa posición de saber sobre un sujeto que no puede hablar por sí mismo, debido a un déficit constitutivo atribuido a su minoría de edad, la psicología forense ocupa el lugar de perito, es decir, el sentido del acto trasgresor, su procedencia y su destino corresponde a determinaciones que el psicólogo devela desde el lugar de un tercero objetivo que posee a su alcance metodológico las herramientas científicas para tal propósito. En esta perspectiva, lo que el niño o el adolescente puedan decir acerca de su acto o de sí mismo, es un material clasificable en una serie de categorías previamente definidas y que sirve a la confirmación o al rechazo de una asignación diagnóstica que se espera explique todo lo que debe saberse del niño o del adolescente trasgresor.

El psicoanálisis se resiste a esta forma de evaluar y clasificar al sujeto. Para el psicoanalista el principio socrático de la docta ignorancia es fundamental tanto en el ejercicio de la investigación de fenómenos sociales, como en la práctica clínica que aplica en este tema. De tal manera, lo primero en reconocer es que solo el sujeto trasgresor es quien tiene la posibilidad de producir un saber que dé cuenta de su acto y del sentido que puede leerse en el mismo, por lo tanto, el trabajo del analista consiste en esta vía en plantear las preguntas y hacer los señalamientos precisos que hagan emerger la producción de este saber.

sujeto a un "Otro" que le inscribe constantemente en una angustia de encuentros y desencuentros. Todo esto permite afirmar que la concepción ontológica que asume el psicoanálisis, se define por el imperativo de un faltante latente y presente en la estructura psíquica de un sujeto que se anuda a la vida, a su cuerpo, a la cultura, a la sociedad y a su propia humanidad por efectos del lenguaje, ya que son los recursos de este último aquellos que le permiten nombrar y hacer algo con dicha falta. 
En este segundo aspecto resulta pertinente señalar la importancia que tiene otorgarle un lugar a la singularidad en el tratamiento del sentido del acto trasgresor. Con la exclusión del sujeto en la evaluación diagnóstica que se implementa desde la psicología forense, se ha llegado a formalizar una serie de protocolos que han conducido a pensar que un acto trasgresor puede leerse como un contenido que se replica en otro acto de la misma naturaleza, o en su defecto, que puede interpretarse a partir de otro acto similar ya acontecido.

Esta estandarización se ha convertido en una manera de blindar ese lugar supuesto saber, en el que se legitima la intervención de la psicología forense en estos temas. Sin embargo, lo que no debe perderse de vista en este asunto es que el propósito a privilegiar radica en la comprensión del acto trasgresor y no en la reivindicación de un campo de aplicación. En consecuencia, es prudente acoger para tal comprensión un análisis que no desconozca la implicación particular del sujeto en materia del carácter singular que dicha implicación imprime sobre el acto en cuestión.

En tercer lugar se encuentra la noción de culpa relacionada con el niño y con el adolescente trasgresor. El contraste que surge entre psicología y psicoanálisis en este punto radica en la discusión de las implicaciones que tiene la conciencia y el inconsciente en la experiencia subjetiva recogida en dicha noción. La psicología asume un concepto de culpa muy diferente al que asume el derecho. En psicología forense la noción de culpa involucra una dimensión cognitiva y afectiva que en la noción homónima del derecho no es contemplado.

La psicología forense sostiene que, en primer lugar, la culpa acontece como un efecto que toma lugar en la conciencia de un sujeto como resultado de un evento desencadenado por este mismo, por lo tanto, sin este evento desencadenante la percepción consiente de un reproche o el sentimiento de culpa es juzgado como irracional, ilógico o producto de una distorsión cognitiva situada en una interpretación errada de la realidad. En segundo lugar, también sostiene esta postura que la culpa es entendida como una asignación de responsabilidad de naturaleza jurídica, que deviene de la pronunciación de una sentencia en la que se ha tomado previa distancia de la posición subjetiva del implicado a ser juzgado al respecto.

El psicoanálisis, por otra parte, asume una posición que subvierte por completo esta lógica en la que opera la psicología y el derecho. En principio se considera a partir de este cuerpo teórico que la culpa puede anteceder al acto trasgresor, incluso puede ésta ser la compulsión que empuje al sujeto a la ejecución de dicho acto o de igual forma, a su reincidencia (Freud, 1916; Tendlarz y Duarte, 2008; Seguí, 2012). La culpa puede anteceder al acto trasgresor 
y esto no obedece a una distorsión o un trastorno de la lógica que sirve al sujeto para interpretar la realidad, esto es absolutamente posible en un sujeto cuya vida anímica, se pone por escenario para que éste construya mediante el lenguaje el sentido y el significante con que nombra y ordena sus vivencias.

Otro asunto relativo al análisis de la culpa en el adolescente y en el niño trasgresor que pone de manifiesto una lectura de orden psicoanalítico, atañe a la comprensión del lugar que tiene el inconsciente y lo inconsciente (Freud, 1923) en la experiencia de la culpa. El hecho de que en un acto trasgresor pueda no estar involucrada la intencionalidad, no es equiparable a que el motivo del mismo sea inconsciente o radique en el inconsciente. Una precisión importante a señalar en el tratamiento de este aspecto aparece como un aporte del trabajo adelantado en el ámbito de la criminología por Jacques Lacan (1966), quien a propósito hace referencia a la implicación que denomina asentimiento subjetivo, la cual define la posición de apropiamiento que hace el sujeto ante sí mismo acerca de las condiciones y consecuencias que le comprometen con su acto trasgresor.

A partir de dicho aporte, es comprensible que en un sujeto pueda haber culpa pero no asentimiento subjetivo, o pueda haber un reproche pero no una posición de responsabilidad subjetiva que le lleve a interrogarse y hacerse cargo de su propio acto ante sí mismo, más que ante una segunda o tercera persona. El asentimiento subjetivo es precisamente aquello que conduce a un saber sobre lo inconsciente, que no puede ser develado por una tercera persona que asuma el lugar de interprete, puesto que cada acto trasgresor tiene una historia particular, una historia que no se encuentra contenida en la razón, en la conciencia de la intencionalidad o en la lectura distante y objetiva de la mirada del otro. Esta es una historia que no a partir de la culpa sino del asentimiento, pugna el sujeto por develar en la lectura de su propio inconsciente.

Sobre la base de estos tres elementos conceptuales, la teoría psicoanalítica se plantea una manera de entender la responsabilidad relativa a la relación entre adolescencia y criminalidad, que resulta ser muy diferente a la concepción de responsabilidad que ha sido formulada en los contenidos de la psicología forense y el derecho penal (Chaves, 2005). Esta formulación de orientación psicoanalítica, es lo que en este trabajo ha tenido lugar en el concepto de responsabilidad subjetiva.

En esta propuesta que brinda el psicoanálisis es importante destacar tres cualidades implícitas en la noción mencionada, ya que dichas cualidades permiten una mejor comprensión no solo de la diferencia sino también 
del alcance que posee esta forma de entender la responsabilidad criminal, llegando más allá de las miradas que ofrecen la psicología y el derecho.

Un primer asunto bastante notable, corresponde a la concepción de sujeto a la cual se dirige el psicoanálisis en relación con el concepto de responsabilidad subjetiva. En esta perspectiva se ha mencionado que el niño y el adolescente son entendidos como sujetos del lenguaje, esto conlleva a que son sujetos capaces de hacer uso de la palabra y, por ende, de las implicaciones psíquicas que esta tiene. Por consiguiente, el niño y el adolescente no son entendidos desde la incapacidad de dar cuenta de su acto transgresor, o desde la necesidad de que un tercero de cuenta por ellos a razón de este acto. En este sentido, la responsabilidad subjetiva asumida como concepto para el análisis de la relación entre adolescencia y criminalidad, implica que el menor de edad no es necesariamente una víctima circunstancial o un instrumento en la ejecución del delito, sino que puede involucrarse en este último en posición de autor en tanto sujeto hablante y anímicamente presente.

El concepto de responsabilidad subjetiva, se dirige entonces al niño y al adolescente como sujetos del derecho y no simplemente como sujetos de derecho. Reconocer la posición de un menor de edad como autor de un acto delictivo, es reconocer que el menor de edad no representa psíquicamente un recipiente vacío; por el contrario, se asume en el niño y en el adolescente una vida anímica suficiente para que dicho sujeto tenga a su alcance las facultades necesarias para sentir la compulsión hacia un acto transgresor $\mathrm{y}$, consecuentemente, elaborar la manera de ejecutar dicho acto. La responsabilidad subjetiva no debe entenderse como una orientación que culpabiliza al menor de edad, pero debe leerse en esta noción el reconocimiento de un sujeto no determinado por su edad o por su ambiente, sino como un sujeto que tiene una posición ante el mundo que lo rodea, ante la norma y ante su manera de responder frente a estos dos últimos (Chaves, 2005).

Otro aspecto que implica el concepto de responsabilidad subjetiva en virtud de sus fundamentos en la teoría psicoanalítica, es que esta noción permite la emergencia de aquello que en psicoanálisis es comprendido como un "sujeto ético" (Gallo, 2007). Se había mencionado que una diferencia entre la psicología forense y el psicoanálisis se encuentra identificada en la manera de interpretar el sentido del acto transgresor del niño o del adolescente, pues bien, en psicoanálisis, un sujeto ético es justamente aquel que elige hacerse cargo de su propio acto sin transferir las consecuencias o interpretación del mismo, a una segunda o tercera persona.

Un último aspecto que define el concepto de responsabilidad subjetiva, pero que también lo diferencia de la noción de responsabilidad aludida en psico- 
logía forense y en derecho penal, está relacionado con la manera en la que el psicoanálisis entiende por su parte la noción de culpa y responsabilidad. Para empezar, es preciso afirmar que en los marcos de la teoría psicoanalítica, la culpa y la responsabilidad son entendidas como experiencias asumidas de manera subjetiva, no delegadas, adjudicadas o imputadas por una segunda o una tercera persona, que para el caso que atañe a este estudio, estas últimas serian un psicólogo interprete o un juez.

Es en este punto donde aparece la importancia del concepto Lacaniano de asentimiento subjetivo. Esta experiencia completamente psíquica implica que la interrogación por la ejecución del acto criminal, e incluso parte del reproche por el mismo, no es producto de una influencia externa, sino producto de un compromiso ético asumido por el sujeto para producir un saber y una posición sobre su acto, independientemente de lo que otros puedan enunciar acerca del acto criminal en cuestión. El asentimiento subjetivo, por tanto, no emerge a partir de una adjudicación que funciona como la responsabilidad penal, es más bien la inquietud de un sujeto que pretende saber algo sobre sí y sobre su acto y ese algo solo puede ser interrogado y contestado desde el propio sujeto.

En la posición de responsabilidad subjetiva no es menester la presencia de la culpa, esta última entendida en tanto psicológica, como jurídicamente. El sujeto movido por el asentimiento subjetivo puede o no encontrarse influenciado por aquello que jurídicamente se reconoce como participación material o intelectual en la ejecución de un delito, también puede o no encontrarse afectado por la influencia de la percepción de su conciencia, en razón de atribuirse la autoría de una infracción legal; en ambas circunstancias, el asentimiento subjetivo opera como una experiencia psíquica que no debe confundirse con la culpa y que no depende de la presencia de esta misma.

El asentimiento subjetivo no es una operación de la conciencia ni un producto de la razón, aunque actúa por medio de la razón y la conciencia esta experiencia psíquica se define en mejores términos como un reclamo del inconsciente, para entender su nexo con un acto que reclama por el conocimiento que le otorga sentido en la historia singular del sujeto. La reflexión desarrollada hasta este punto ha dejado claro que la noción de responsabilidad subjetiva se ha construido a partir de referentes propios del psicoanálisis y bastante diferentes de aquellos asumidos en la psicología forense y el derecho. En consecuencia, esta propuesta de orientación psicoanalítica no se limita a las cuestiones que acontecen solo en el límite práctico de los tribunales judiciales, sino que tiene mucho que ofrecer al análisis del vínculo político que teje la relación entre adolescencia y criminalidad. 


\section{Conclusiones}

Los análisis desarrollados a lo largo de esta investigación corresponden en su totalidad al dialogo que desde la perspectiva de la ciencia política, definida en las directrices conceptuales del profesor Alcántara, son posibles de plantear con la filosofía Kantiana, el psicoanálisis, la teoría del derecho y los aportes metodológicos del filósofo francés Michel Foucault.

Las discusiones jurídicas radican en el acontecer de la jurisprudencia, y asimismo en la aplicación de la ley sobre las decisiones que debe tomar un estrado judicial en casos específicos. En una reflexión diferente a tal lineamiento jurídico, la discusión que ha tenido lugar en este estudio ha implicado el análisis sobre la manera en que durante los últimos veintiséis años el sistema de gobierno que ha tenido vigencia en Colombia, ha construido y sostenido sus políticas de infancia y adolescencia. Por esta razón han sido pertinentes las categorías de análisis propuestas por el profesor Alcántara como Polity, Policy y Politics.

Las conclusiones que responden a la pregunta de investigación comienzan por precisar que el niño y el adolescente al que se dirige el SRPA, es un sujeto reconocido mediante una categoría política. Cuando la Ley 1098 de 2006 alude al menor de edad, no se refiere al niño o al adolecente en tanto su complejidad psicológica o sociológica, sino a la denominación política que según las directrices constitucionales ha sido atribuida a este sujeto por disposición del Estado colombiano (Procuraduría General de la Nación, 2006).

La categoría política denominada minoría de edad, enuncia una posición de vulnerabilidad en la que se encuentran definidos por el Estado colombiano los niños y los adolescentes. Con base en los conceptos de la filosofía kantiana, que son por su parte los conceptos que fundamentan las políticas de las sociedades liberales, un menor de edad es entendido como un sujeto incapaz de responder por sí mismo, privado de autonomía y desprovisto del criterio suficiente para hacer sus propios juicios.

La asignación legislativa de dicha categoría a niños y adolescentes les sitúa en un sector de la sociedad civil donde no son reconocidos como sujetos del derecho, sino como sujetos de derecho. Por esta razón, gozan de las prerrogativas que provee el Estado, pero se ven imposibilitados de participar en los mecanismos de deliberación pública y, bajo ninguna circunstancia, son considerados como objetos punitivos para las autoridades judiciales (Hernández Basualto, 2007; Huertas Díaz y Morales Chinome, 2013). 
La noción de niño y de adolescente que opera en el SRPA con base en la Ley 1098 de 2006, se encuentra entonces formulada dentro de las condiciones del concepto de "minoría de edad". Ahora bien, lo que no resulta explícito en los documentos estatales al respecto es que las implicaciones de funcionar a partir de esta noción no son solo políticas y legislativas, son también económicas e inciden en las dinámicas de las relaciones internacionales.

El Estado colombiano se ha vinculado constitucionalmente desde el año de 1991 a las políticas de la ONU dedicadas a la protección de los derechos del niño. Visibilizar iniciativas que corroboren este compromiso favorece la posición política de Colombia para participar de otros tratados internacionales relacionados con apoyo económico e intercambio de beneficios diplomáticos y mercantiles. Por esta razón, el Sistema colombiano de Responsabilidad Penal para Adolescentes, ha asumido una concepción de vulnerabilidad asociada al menor de edad que ha resultado consistente con la noción de vulnerabilidad asociada por la ONU a los niños, niñas y adolescentes latinoamericanos (ONU, 2010). Algo similar ocurre con las explicaciones que relacionan en Colombia la relación entre adolescencia y criminalidad.

$\mathrm{Al}$ interrogar las explicaciones que relacionan la adolescencia y la criminalidad según las disposiciones del SRPA, ha sido posible encontrar entre tantas teorías que proveen dichas explicaciones, un sistema legislativo que a conveniencia excluye algunas de esas teorías, mientras promueve el auge de otras. En este orden de ideas, las teorías que sirven al sostenimiento de la categoría política enunciada como "minoría de edad", encuentran mayor difusión que aquellas que proponen un cambio en la forma de entender al niño y al adolescente.

Entre las teorías con mayor aceptación institucional de parte del Estado colombiano, figuran principalmente aquellas que operan en tendencias de carácter biologicista y ambientalista. Otras teorías que plantean la posibilidad pedagógica de un niño y un adolescente con capacidad y juicio para responder por una infracción social o legal, son notoriamente excluidas del panorama político y legislativo en consideración; entre tales propuestas destacan aquellas de fundamentación socio-cultural (Vygotski, 1978; Rodríguez Arocho, 1999), y también las de fundamentación en procesamiento cognitivo (Palomino Gonzales, 1989; Lind, 1999).

El criterio que discrimina la inclusión o exclusión de estas teorías como fundamento del SRPA, no obedece al poder explicativo o los avances científicos y la vigencia propios de las mismas. Según ha logrado identificarse mediante esta investigación, dicha discriminación obedece a los intereses que puede tener un sistema de gobierno determinado, para legislar la administración 
publica en función de propósitos revelados mediante la categoría de análisis definida como Politics.

En este sentido, las teorías de orientación biologicista y ambientalista ocupan un lugar predominante, en las lógicas que vinculan en el SRPA las nociones de adolescencia y criminalidad. Sin embargo, estas teorías no sugieren un mayor poder explicativo o un avance considerable en relación a teorías cognitivas, socio-culturales o de orientación psicoanalítica. Lo que sí suele acontecer, es que las teorías dominantes resultan más funcionales para efectos de los manejos presupuestales que tienen lugar en las políticas de infancia y adolescencia, en las cuales no es extraño encontrar casos de malversación de fondos (El Heraldo, 2016).

Como puede notarse en este punto, enunciados que definen la infancia como población vulnerable o la adolescencia como una etapa de alta sensibilidad a la criminalidad, no son el resultado de investigaciones científicas exhaustivas. Estos enunciados, según ha sido posible señalar en esta investigación, son el producto de un discurso a través del cual un sistema de gobierno se asegura la gobernanza, con el propósito de sostener un estatu quo que oculte los componentes de una Politics, bajo el funcionamiento de una Policy y la legislación de una Polity.

A la luz de esta investigación, en la relación entre adolescencia y criminalidad existen maneras de comprender al sujeto implicado que son diferentes a como éste es asumido en el SRPA. Si bien el mencionado sistema se plantea la exigencia de exonerar al menor de edad del cargo de responsabilidad penal, deja a su vez abierta la posibilidad de proponer la noción de "responsabilidad subjetiva" en relación al niño o el adolescente trasgresor.

Las aproximaciones propuestas desde el psicoanálisis a la comprensión del sujeto criminal, ofrecen elementos teóricos y metodológicos que brindan lugar a la emergencia de un sujeto ético en esa relación entre adolescencia y crimen (Gallo, 2007). Hacer referencia a un sujeto ético en esta perspectiva, es aludir a un sujeto que elige hacerse cargo de su acto trasgresor no solo para asumir las consecuencias del mismo, sino también para producir un saber que no puede ser develado por una segunda o tercera persona, acerca de la naturaleza, el sentido y la función susceptibles de indagar en su proceder criminal.

En el marco de esta reflexión aparece el concepto de responsabilidad subjetiva, un concepto aportado por el psicoanálisis, cuya base corresponde con la manera en que este cuerpo teórico y metodológico se plantea una concepción de sujeto y una explicación sobre la experiencia de la culpa y la experiencia del sentido del acto trasgresor. El concepto de responsabilidad subjetiva 
comporta unos lineamientos muy diferentes a los que conlleva el concepto de responsabilidad penal, en consecuencia, es posible afirmar que si bien un adolescente puede ser eximido de la responsabilidad penal que sigue a un quebrantamiento de la ley, no implica esto que en dicho adolescente no aparezca una posición ética de responsabilidad subjetiva.

El recorrido implicado en esta investigación y sus respectivos hallazgos conservan una relación de consistencia con el marco de antecedentes consignado en este documento. En dicho apartado quedó registrada la insistencia del Profesor Berríos (2005, 2011), entre otros investigadores, en la necesidad de ocuparse del análisis relativo a la inimputabilidad atribuida al adolescente en función exclusiva de su minoría de edad, ya que los argumentos y las discusiones a este respecto han sido insuficientes en lo que concierne por lo menos a las legislaciones de Chile, Argentina y Colombia. El estudio del que da cuenta el presente proyecto aporta las especificaciones de algunos hallazgos orientados por el interés de profundizar en las cuestiones que señala el investigador citado.

\section{Referencias}

Aguilar, L. (2014). La nueva Gobernanza Pública. Conferencia pronunciada el 25 de Marzo de 2014 en el Centro de Gobernanza Pública y Corporativa de la Universidad de Turabo, para la cátedra designada como La Gobernanza de los Asuntos Públicos. Puerto Rico. Disponible en http://ut.suagm.edu/sites/default/ files/uploads/Centro-Gobernanza/CATEDRAL_ANUAL/La-Nueva-GobernanzaPublica.PR.UT.pdf

Alarcón, R., Vidal, G. (1986). Psiquiatría. Editorial Médica PANAMERICANA S.A. Argentina.

Alcántara Sáez, M. (1993). Cuando hablamos de ciencia política, ¿De qué hablamos? Revista Mexicana de Sociología. UNAM. LV.4. Octubre-Diciembre. México.

Berríos Díaz, G. (2011). La Ley de Responsabilidad Penal del Adolescente como Sistema de Justicia: Análisis y propuestas. Boletín Electrónico Política Criminal, 6 (11), 163-191. Disponible en: http://www.politicacriminal.cl/Vol_06/n_11/ Vol6N11A6.pdf

Berríos Díaz, G. (2005). El nuevo sistema de justicia penal para adolescentes. Revista de Estudios de la Justicia, 6, pp. 1-15, DOI: 10.5354/0718-4735.2011.15079 
Casco, F., Oliva, A. (2004). Ideas sobre adolescencia entre padres, profesores, adolescentes y personas mayores. Revista Apuntes de Psicología, 22 (2), 171-185. URI: http://hdl.handle.net/11441/13995

Corte Constitucional de Justicia. (2000). Ley 599 del 2000. Código de Procedimiento Penal Colombiano. Disponible en: http://oig.cepal.org/sites/default/files/2000_ codigopenal_colombia.pdf

Consejo Superior de la Judicatura. (S.F). ABC del Sistema de Responsabilidad Penal para Adolescentes, esquema operacional y catálogo de audiencias. Documento Estatal Informativo publicado y difundido por la Rama Judicial del Consejo Superior de la Judicatura. Bogotá, Colombia. Disponible en:https://www.ramajudicial.gov.co/ documents/10228/1559849/Contenido+Sistema+Penal+ para+Adolescentes. pdf/08ff6d1e-21c4-40d0-a77c-947679157158

Congreso de la Republica de Colombia. (2006). Ley 1098 de 2006. Versión revisada y actualizada a Noviembre de 2016. Disponible en: http://www.secretariasenado. gov.co/senado/basedoc/ley_1089_2006.html

Comité Operativo Distrital de Infancia y Adolescencia. (2011). La Política de Infancia y Adolescencia en Bogotá D.C 2011-2021. Informe publicado por la Alcaldía Mayor de Bogotá D.C. Colombia. Disponible en: http://www.idrd.gov.co/sitio/ $\mathrm{idrd} /$ sites/default/files/imagenes/POLITICA\%20DE\%20INFANCIA\%20Y\%20 ADOLESCENCIA\%20TODO.pdf

Chaves, G. (2005). Preguntar al psicoanálisis por la responsabilidad del sujeto. Revista Desde el Jardín de Freud, 5: 286-303. Disponible en https://dialnet.unirioja.es/ descarga/articulo/2922528.pdf

Equipo de Investigación Proyecto Colombia Nunca Más. (2008). Colombia Nunca Más: Crímenes de Lesa Humanidad en la zona quinta. Informe publicado por la Corporación Colectivo de Abogados “José Alvear Restrepo". Bogotá D.C. Colombia.

Escobar, M. (2013). Extractos de jurisprudencia: Sistema de Responsabilidad Penal para Adolescentes, Ley 1098 de 2006: Menor Infractor. Documento de Estado publicado en Marzo de 2013 por la Sala de Casación Penal de la Corte Suprema de Justicia. República de Colombia.

El Heraldo, (2016). Fiscalía captura a 11 contratistas del ICBF en La Guajira. Noticia publicada en el diario de difusión vía internet titulado El Heraldo. 20 de Octubre de 2016-06:03. Disponible en https://www.elheraldo.co/la-guajira/once-capturasen-la-guajira-por-hechos-de-corrupcion-en-el-icbf-295509

Freud, S. (1930). El Malestar en la cultura. Tomado del Tomo III de la cuarta edición de las Obras Completas de Sigmund Freud publicada en 1981. Versión traducida 
al castellano por Luis López- Ballesteros y de Torres. Editorial Biblioteca Nueva. Madrid, España. p. 3017-3067.

Freud, S. (1923). El $<Y O>$ y el $<E L L O>$. Tomado del Tomo III de la cuarta edición de las Obras Completas de Sigmund Freud publicada en 1981. Versión traducida al castellano por Luis López- Ballesteros y de Torres. Editorial Biblioteca Nueva. Madrid, España. p. 2701 - 2728.

Freud, S. (1916). Varios tipos de carácter descubiertos en la labor analítica. Tomado del Tomo III de la cuarta edición de las Obras Completas de Sigmund Freud publicada en 1981. Versión traducida al castellano por Luis López- Ballesteros y de Torres. Editorial Biblioteca Nueva. Madrid, España. p. 2413-2428.

Foucault, M. (1969). La Arqueología del Saber. Vigesimotercera edición en español de 2007. Siglo XXI editores S.A. México D.F.

Foucault, M. (1970). El Orden del Discurso. Traducción al castellano de 1973 de Alberto Gonzales Troyano. Quinta edición de 2010 de Fabula TusQuets Editores. Barcelona, España.

Foucault, M (1994). ¿Qué es la Ilustración? Tomado de la publicación de Estética, Ética y Hermenéutica. De la traducción al castellano de Ángel Gabilondo. De la edición en castellano de 1999 publicada por la Editorial Paidós Ibérica S.A. Barcelona. p. 335-352.

Gallo, H. (2007). El Sujeto Criminal: una aproximación psicoanalítica al crimen como objeto social. Tomado de la colección Psicoanálisis, Sujeto y Sociedad. Medellín: Editorial de la Universidad de Antioquia.

Gallo, H., Ramírez, M. (2012). El Psicoanálisis y la investigación en la universidad. Buenos Aires: Grama Ediciones.

Hernández Basualto, H. (2007). El nuevo derecho penal de adolescentes y la necesaria revisión de su "teoría del Delito". Revista de Derecho, 2 (XX), 195-217. Disponible en http://www.redalyc.org/articulo.oa?id=173714174009

Huertas Díaz, O., Morales Chinome, N. (2013). El sistema de responsabilidad penal para adolescentes: la expansión de la punibilidad en el neopunitivismo colombiano. Revista Científica Guillermo de Ockham. 11 (2), 69-78, Disponible en https://dialnet.unirioja.es/descarga/articulo/4607390.pdf.

Jara, M., Ferrer, A. (2005). Genética de la violencia. Revista Chilena de Neuropsiquiatría. 43 (3), 188-200. http://dx.doi.org/10.4067/S0717-92272005000300003

Kant, I. (1784). Respuesta a la pregunta: ¿Qué es la Ilustración? Disponible en https:// geografiaunal.files.wordpress.com/2013/01/kant_ilustracion.pdf 
Lacan, J. (1966). Escritos 1. Tomado de la décima edición en español publicada en 1984. Versión corregida y aumentada traducida por Tomás Segovia. Madrid: Siglo XXI Editores Internacional.

Lind, G. (1999). Una introducción al test del juicio moral. Tomado de la traducción al castellano de Manuel José Salazar. Publicado por el Departamento de Psicología de la Universidad de Konstanz, Alemania.

Ministerio de Desarrollo Social de la Nación; UNICEF \& Universidad Nacional de Tres de Febrero. (2008). Adolescentes en el Sistema Penal: Situación actual y propuestas para un proceso de transformación. Documento Estatal publicado y difundido por las organizaciones que responden de su autoría. Buenos Aires, Argentina. Consultado en: http://www.unicef.org/argentina/spanish/Adolescentes_en_el_ sistema_penal.pdf

Organización de Naciones Unidas (ONU). (2010). Pobreza Infantil en Latinoamérica y el Caribe. Informe publicado por la Organización de las Naciones Unidas mediante las editoriales de UNICEF y CEPAL en Diciembre de 2010. Consultado en: https:// www.unicef.org/honduras/Pobreza_infantil_America_Latina_Caribe_2010.pdf

Palomino Gonzales, A. (1989). Laurence Kohlberg: teoría y práctica del desarrollo moral en la escuela. Revista interuniversitaria de formación del profesorado, 4: 79-90. Disponible en: file:///C:/Users/Usuario/Downloads/DialnetLaurenceKohlberg-117615\%20(3).pdf

Perinat, A. (1998). Psicología del Desarrollo: Un enfoque sistémico. Barcelona: Ediciones EDIUOC de la Universidad Oberta de Cataluña. Cap. 24.

Procuraduría General de la Nación. (2006). Ley 1098: Ley de Infancia y de la Adolescencia. Versión autorizada editada y promovida por VISION MUNDUAL. República de Colombia.

Rodríguez Arocho, W. (1999). La relación desarrollo-aprendizaje en las teorías de Jean Piaget y Lev S. Vygotski, Un análisis comparativo. Acta Colombiana de Psicología, 2, 29-37, Disponible en https://vdocuments.site/rodriguez-arocho-w1999-la-relacion-desarrollo-aprendizaje

Roll, D. (2011). La Política al Diván: las explicaciones psicológicas de la política en Freud, Fromm y Marcuse. Medellín: Editorial de la Escuela de Derecho y Ciencias Políticas de la Universidad Pontificia Bolivariana.

Skinner. B.F. (1971). Más allá de la libertad y la dignidad. Traducción al castellano de Juan José Coy. Primera edición de 1972, Barcelona: Editorial Fontanella S.A.

Skinner, B.F. (1974). Sobre conductismo. De la traducción al castellano de Fernando Barrera. Barcelona: Edición internacional de 1987 de Martínez Roca S.A. 
Sanders, D. (1995). El análisis conductista. En David Marsh y Gerry Stoker (edit). Teoría y Métodos de la Ciencia Política. Tomado de la traducción al castellano de Jesús Cuéllar Menezo. Madrid: Alianza Editorial. Cap. 3. p. 69-84.

Seguí, L. (2012). Sobre la responsabilidad criminal: psicoanálisis y criminología. Madrid: Fondo de Cultura Económica de España.

Soria, M.A. (1998). Psicología y práctica jurídica. Barcelona: Ed. Ariel S.A.

Tendlarz. S., Duarte, C. (2008). A quién mata el asesino. Buenos Aires: GRAMA Ediciones.

Vygotski, L. (1978). El desarrollo de los procesos psicológicos superiores. Barcelona: Editorial CRÍTICA S.A. Segunda Parte. Cap. VI. p. 123-140. 